\title{
PREVALÊNCIA E PERFIL DAS PESSOAS COM DIABETES CADASTRADAS NO SISTEMA DE INFORMAÇÃO DA ATENÇÃO BÁSICA (SIAB)
}

\author{
Prevalence and profile of people with diabetes registered at the \\ primary care information system (SIAB)
}
Prevalencia y perfil de personas con diabetes registradas en el Sistema de Información de la Atención Básica (SIAB)

Artigo Original

\section{RESUMO}

Objetivo: Avaliar a prevalência e o perfil das pessoas com diabetes mellitus (DM), autorreferidas. Métodos: Estudo transversal, retrospectivo, realizado com os dados secundários extraídos do Sistema de Informação da Atenção Básica (SIAB). Os dados foram obtidos junto à Secretaria Municipal de Saúde nos meses de março a abril de 2014. Coletaram-se os dados do SIAB referentes à população com diabetes autorreferidas (com 15 anos ou mais) do município de Lajeado-RS, no período de 2011 a 2013, analisados através de estatística descritiva, utilizando-se o programa SPSS versão 21. Resultados: Verificou-se que a prevalência das pessoas com DM se manteve equiparada nos anos estudados $(3,0 \%$ em $2011,3,1 \%$ em 2012 e 3,0\% em 2013). Essa população, na sua maioria, estava acima de 60 anos (60\% em 2011, 58\% em 2012 e 60\% em 2013), é alfabetizada ( $88 \%$ em 2011, 89,9\% em 2012 e $90,7 \%$ em 2013), com predominância do sexo feminino (63,2\% em 2011, 62,9\% em 2012 e $63,7 \%$ em 2013) e hipertensa (77,5\% em $2011,76,1 \%$ em 2012 e $76,9 \%$ em 2013$)$. A prevalência de gestantes com diabetes foi de $0,5 \%$ em 2012 e $0,3 \%$ em 2013, não havendo casos em 2011. Conclusão: A prevalência de DM autorreferida no município é em torno de $3 \%$ nos anos analisados, abaixo da média nacional. Constatou-se que a maioria da população com DM é idosa, feminina, alfabetizada, hipertensa, além de haver baixa prevalência de DM em gestantes.

Descritores: Diabetes Mellitus; Prevalência; Saúde Pública; Gestão em Saúde.

\section{ABSTRACT}

Objective: To assess the prevalence and profile of people with self-reported diabetes mellitus (DM). Methods: A retrospective cross-sectional study conducted with secondary data extracted from the Sistema de Informação da Atenção Básica - SIAB (Primary Care Information System). Data were obtained from the Municipal Health Secretariat during March and April 2014. SIAB data collected referred to the population with self-reported diabetes (15 years and older) of the municipality of Lajeado, RS, for the period between 2011 and 2013 and underwent descriptive statistical analysis using the SPSS 21.0 software. Results: We found that the prevalence of people with DM remained the same throughout the years studied (3.0\% in 2011, 3.1\% in 2012, and 3.0\% in 2013). This population was mostly aged over 60 (60\% in 2011, 58\% in 2012, and 60\% in 2013), literate (88\% in 2011, 89.9\% in 2012, and 90.7\% in 2013), and predominantly female (63.2\% in 2011, 62.9\% in 2012, and $63.7 \%$ in 2013) and hypertensive (77.5\% in 2011, 76.1\% in 2012, and $76.9 \%$ in 2013). The prevalence of pregnant women with diabetes was of $0.5 \%$ in 2012 and of $0.3 \%$ in 2013, with no cases in 2011. Conclusion: The prevalence of self-reported DM in the municipality is around 3\% for the years analyzed, which is below the national average. It was found that the majority of the population with DM is older, female, literate and hypertensive, and there is a low prevalence DM among pregnant women.

\section{Claudete Moreschi ${ }^{(1)}$ \\ Claudete Rempel ${ }^{(1)}$ Ioná Carreno ${ }^{(1)}$ \\ Daniel Silveira da Silva ${ }^{(1)}$ \\ Carmen Neri Fernández Pombo $^{(2)}$ \\ María Reyes Luna Cano ${ }^{(2)}$}

1) Centro Universitário UNIVATES Lajeado (RS) - Brasil

2) Universidad de Vigo - Vigo - Espanha

Recebido em: 24/04/2015 Revisado em: 14/06/2015 Aceito em: 25/06/2015

Descriptors: Diabetes Mellitus; Prevalence; Public Health; Health Management. 


\section{RESUMEN}

Objetivo: Evaluar la prevalencia y el perfil de las personas con Diabetes Mellitus (DM) autorreferidas. Métodos: Estudio transversal y retrospectivo realizado con datos secundarios del Sistema de Información de la Atención Básica (SIAB). Los datos fueron obtenidos en la Secretaria Municipal de la Salud entre los meses de marzo y abril de 2014. Se recogieron datos del SIAB concerniente ala población con diabetes autorreferida (de 15 años o más) del municipio de Lajeado-RS en el periodo entre 2011 y 2013 analizados a través de la estadística descriptiva y la utilización del programa SPSS versión 21. Resultados: Se verifico que la prevalencia de las personas con DM se mantuvo parecidas en los años investigados (el 3,0\% en 2011, el 3,1\% en 2012 y el 3,0\% en 2013). Esta población, en su mayoría, tenía más de 60 años (el 60\% em 2011, el 58\% en 2012 y el 60\% en 2013), está alfabetizada (el 88\% en 2011, el 89,9\% en 2012 y el 90,7\% en 2013), con predominancia del sexo femenino (el 63,2\% en 2011, el $62,9 \%$ en 2012 y el 63,7\% en 2013) e hipertensa (el 77,5\% en 2011, el 76,1\% en 2012 y el 76,9\% en 2013). La prevalencia de embarazadas con diabetes fue del $0,5 \%$ en 2012 y del $0,3 \%$ en 2013, sin casos en 2011. Conclusión: La prevalencia de la DM autorreferida en el municipio es del 3\% en los años investigados lo que está por debajo de la media nacional. Se constató que la mayoría de la población con DM es mayor, mujer, alfabetizada, hipertensa, además de tener baja prevalencia de DM en las embarazadas.

Descriptores: Diabetes Mellitus; Prevalencia; Salud Pública; Gestión en Salud.

\section{INTRODUÇÃO}

A prevalência de diabetes melittus (DM) cresce progressivamente, tornando esse distúrbio endócrino um problema de saúde pública com proporções epidêmicas ${ }^{(1)}$. Dados recentes da International Diabetes Federation (IDF) apontam que, no ano de 2013, o diabetes provocou 5,1 milhões de mortes e custou mais de 548 bilhões de dólares aos serviços de saúde ${ }^{(2)}$.

A faixa etária predominante encontra-se entre 40 e 59 anos, e apesar de atingir todas as classes sociais, $80 \%$ das pessoas com diabetes vivem em países subdesenvolvidos ou em desenvolvimento. O Brasil ocupa a quarta posição no ranking dos países com maior número de pessoas com diabetes entre 20 e 79 anos, tendo 11,9 milhões de pessoas com a doença ${ }^{(2)}$. Essa doença acomete $5,6 \%$ da população adulta brasileira, e, em grande parte dessa população, não é diagnosticada antes das suas manifestações crônicas terem-se estabelecido ${ }^{(3)}$, representando, em razão disso, um importante problema de saúde pública para o país ${ }^{(4)}$.

A elevada prevalência do DM é decorrente de diversos fatores, como maior expectativa de vida, sedentarismo, obesidade e urbanização(5). As complicações agudas e crônicas do diabetes causam alta morbimortalidade, acarretando altos custos para os sistemas de saúde. Gastos mundiais relacionados ao diabetes, em 2010, foram estimados em $11,6 \%$ do total dos gastos com atenção em saúde ${ }^{(2)}$

O DM e a hipertensão arterial sistêmica (HAS) são responsáveis pela primeira causa de mortalidade e de hospitalizações no Sistema Único de Saúde (SUS), e representam, ainda, mais da metade do diagnóstico primário em pessoas com insuficiências renais crônicas submetidas à diálise $^{(6)}$.

O controle metabólico aliado a ações de prevenção e tratamento pode anteparar ou retardar o surgimento de complicações crônicas provenientes do $\mathrm{DM}^{(5)}$.

O Sistema de Informação da Atenção Básica (SIAB) é composto por vários questionários aplicados pela Equipe de Saúde da Família (ESF), e as respostas registradas são de autorreferência das pessoas, possuindo importante papel nesse processo, visto que possibilita a realização de levantamento epidemiológico dos indivíduos com diabetes cadastrados na atenção primária. É um facilitador dos processos de trabalho na ESF, subsidiando melhorias e decisões mais precisas na área da saúde ${ }^{(7)}$, o que possibilita a visualização do perfil epidemiológico e sanitário da população de sua área de abrangência, oferecendo um diagnóstico da situação de saúde da população. Além disso, facilita a coleta de dados da população cadastrada na ESF, e os dados podem e devem ser utilizados para planejamentos das ações de saúde ${ }^{(8)}$.

A análise epidemiológica de pessoas com DM mostram dados que possibilitam a implantação de políticas públicas de saúde, visando diminuir as dificuldades dessas pessoas e de suas famílias, e propiciar melhorias à sua qualidade de vida $(\mathrm{QV})^{(5)}$. Tendo em vista que o DM pode provocar impactos desfavoráveis na QV dos indivíduos acometidos, como condição crônica na vida de pessoas, nas famílias e na sociedade ${ }^{(9,5)}$ o levantamento de dados e análise sobre essa doença é de fundamental importância ${ }^{(4)}$.

Assim, o presente estudo tem por objetivo avaliar a prevalência e o perfil das pessoas com diabetes mellitus (DM), autorreferidas.

\section{MÉTODOS}

Trata-se de um estudo transversal, retrospectivo, realizado no município de Lajeado-RS, em que foram analisados os dados dos pacientes de 2011 a 2013 com $\mathrm{DM}$, cadastrados no SIAB. Como critério de inclusão no estudo, era necessário o paciente ter 15 anos ou mais, independentemente de sexo, e seus questionários no SIAB estarem preenchidos corretamente. Os dados foram obtidos 
junto à Secretaria Municipal de Saúde nos meses de março a abril de 2014.

O município de Lajeado encontra-se na região do Vale do Taquari, região central do estado do Rio Grande do Sul - Brasil. Esse município possui 71.445 habitantes, dentre os quais 71.180 são moradores da zona urbana e 265 da zona rural, o que caracteriza o município com alto grau de urbanização $(99,9 \%)^{(10)}$. Segundo o Instituto Brasileiro de Geografia e Estatística (IBGE), em 2013, a população de Lajeado era de 76.187 habitantes ${ }^{(11)}$.

As variáveis coletadas do sistema foram: prevalência da DM, sexo, alfabetização, idade, faixa etária, associação da diabetes com HAS e associação da DM com a gestação, no caso das mulheres.

Aplicou-se a análise descritiva dos dados através do programa SPSS versão 21, apresentados na forma de frequência relativa (\%), e a idade foi apresentada na forma de média ( \pm desvio padrão).

Este estudo seguiu os preceitos da Resolução 466/12 do Conselho Nacional de Saúde, que regulamenta as pesquisas com seres humanos, sendo aprovado através do parecer $n^{\circ}$ 231.467. A utilização dos dados registrados no SIAB teve autorização da Secretaria de Saúde do município de Lajeado-RS, mediante assinatura da Carta de Anuência. Os pesquisadores coletaram e analisaram os dados de forma ética e respeitosa em relação ao município e ao conhecimento científico, conforme Termo de Cessão de Uso de Informação.

\section{RESULTADOS}

A população com 15 anos ou mais do município de Lajeado, cadastrada no SIAB, em 2011, foi de 28.794; em 2012, foi 36.662; e em 2013, foi de 37.495. A prevalência de pessoas com DM castradas no SIAB, na amostra deste estudo, nos anos de 2011 a 2013 foi de 3,0\% $(n=850)$ em 2011, 3,1\% ( $n=1.131)$ em 2012 e 3,0\% $(n=1.131)$ em 2013 (Tabela I).
Com relação ao sexo, nos três anos estudados, a maioria da população cadastrada era mulher, sendo $63,2 \%(n=537)$, $62,9 \%(n=708)$ e $63,7 \%(n=721)$ nos anos 2011, 2012 e 2013 , respectivamente. A média de idade da população foi de $61,7( \pm 13,3)$ em 2011, 61,2 ( $( \pm 13,8)$ em 2012 e 62,0 $( \pm 14,0)$ anos em 2013. Quanto ao nível de escolaridade, o percentual de alfabetizados foi de $88 \%(\mathrm{n}=748)$ em 2011, $89,9 \%(n=1.017)$ em 2012 e 90,7\% $(n=1.026)$ em 2013 (Tabela I).

$\mathrm{Na}$ análise da população das pessoas com DM por faixa etária, observou-se que nos três anos estudados a sua prevalência aumentou nos cadastros entre 15 e 49 anos. Há um grande aumento na prevalência a partir dos 60 anos de idade, tendo maior prevalência de pessoas com DM entre 60 e 69 anos de idade (Tabela II).

Com relação à faixa etária por sexo, observou-se que, em 2011, a maioria da população com DM entre 15 e 49 anos de idade é de homens, mas a partir dos 50 anos a prevalência é maior em mulheres. Em 2012 e 2013, a maioria da população com DM entre 15 e 29 anos de idade é de homens, e a partir dos 30 anos, a maioria da população com DM é de mulheres. A partir dos 90 anos, há alta prevalência do sexo feminino (Tabela II).

Quanto ao número de indivíduos com DM e HAS acima de 15 anos de idade, observou-se que o percentual de pessoas com as duas doenças conjugadas é sempre maior do que de pessoas que possuem apenas DM. Houve uma prevalência de 77,5\% $(n=659)$ em 2011, 76,1\% $(n=861)$ em 2012 e 76,9\% (n=870) em 2013 de indivíduos diabéticos hipertensos (Figura 1).

Ao analisar a prevalência da HAS por faixa etária na população diabética de Lajeado-RS, verificou-se que, nos anos de 2012 e 2013, houve casos de pessoas com diabetes e hipertensão, desde a faixa etária de 15 a 19 anos. Percebeuse também que houve aumento no percentual de pessoas com DM que apresentam HAS à medida que aumenta a faixa etária, nos três anos estudados (Tabela III).

Tabela I - População, com 15 anos ou mais, cadastrada no SIAB de Lajeado-RS, e perfil das pessoas com diabetes mellitus desta população. Lajeado-RS, 2011 a 2013.

\begin{tabular}{|c|c|c|c|c|c|c|}
\hline \multirow{2}{*}{ Ano } & \multirow{2}{*}{$\begin{array}{c}\text { População } \\
\text { SIAB } \\
\text { (15 anos ou mais) }\end{array}$} & \multirow{2}{*}{$\begin{array}{l}\text { Diabéticos } \\
\text { (n) } \%\end{array}$} & \multicolumn{2}{|c|}{$\begin{array}{l}\text { Sexo } \\
\text { (n) } \% \\
\end{array}$} & \multirow{2}{*}{$\begin{array}{l}\text { Idade Média } \\
\text { (DP) }\end{array}$} & \multirow{2}{*}{$\begin{array}{c}\text { Alfabetizados } \\
\text { (n) } \%\end{array}$} \\
\hline & & & $\mathbf{M}$ & $\mathbf{F}$ & & \\
\hline 2011 & 28.794 & $850(3,0)$ & $313(36,8)$ & $537(63,2)$ & $61,7(13,3)$ & $748(88,0)$ \\
\hline 2012 & 36.662 & $1.131(3,1)$ & $423(37,4)$ & $708(62,6)$ & $61,2(13,8)$ & $1.017(89,9)$ \\
\hline 2013 & 37.495 & $1.131(3,0)$ & $410(36,3)$ & $721(63,7)$ & $62,0(14,0)$ & $1.026(90,7)$ \\
\hline
\end{tabular}

M=Masculino; F=Feminino; DP=Desvio padrão; SIAB= Sistema de Informação da Atenção Básica 
Tabela II - Prevalência, por faixa etária e sexo, da população com diabetes mellitus de Lajeado-RS, com 15 anos ou mais, cadastrada no SIAB. Lajeado-RS, 2011 a 2013.

\begin{tabular}{|c|c|c|c|c|c|c|c|c|c|}
\hline \multirow{4}{*}{$\begin{array}{l}\text { Faixa } \\
\text { etária }\end{array}$} & \multicolumn{3}{|c|}{2011} & \multicolumn{3}{|c|}{2012} & \multicolumn{3}{|c|}{2013} \\
\hline & Masculino & Feminino & Total & Masculino & Feminino & Total & Masculino & Feminino & Total \\
\hline & $\mathbf{n}$ & $\mathbf{n}$ & $\mathbf{n}$ & $\mathbf{n}$ & $\mathbf{n}$ & $\mathrm{n}$ & $\mathbf{n}$ & $\mathbf{n}$ & $\mathbf{n}$ \\
\hline & $\%$ & $\%$ & $\%$ & $\%$ & $\%$ & $\%$ & $\%$ & $\%$ & $\%$ \\
\hline & 3 & 1 & 4 & 4 & 3 & 7 & 4 & 4 & 8 \\
\hline $15-19$ & $(75,0)$ & $(25,0)$ & $(0,5)$ & $(57,1)$ & $(42,9)$ & $(0,6)$ & $(50,0)$ & $(50,0)$ & $(0,7)$ \\
\hline $20-29$ & $\begin{array}{c}11 \\
(61,1)\end{array}$ & $\begin{array}{c}7 \\
(38,9)\end{array}$ & $\begin{array}{c}18 \\
(2,1)\end{array}$ & $\begin{array}{c}18 \\
(62,1)\end{array}$ & $\begin{array}{c}11 \\
(37,9)\end{array}$ & $\begin{array}{c}29 \\
(2,6)\end{array}$ & $\begin{array}{c}7 \\
(63,0)\end{array}$ & $\begin{array}{l}10 \\
(37)\end{array}$ & $\begin{array}{c}27 \\
(2,4)\end{array}$ \\
\hline $30-39$ & $\begin{array}{c}11 \\
(45,8)\end{array}$ & $\begin{array}{c}13 \\
(54,2)\end{array}$ & $\begin{array}{c}24 \\
(2,8)\end{array}$ & $\begin{array}{c}15 \\
(37,5)\end{array}$ & $\begin{array}{c}25 \\
(62,5)\end{array}$ & $\begin{array}{c}40 \\
(3,5)\end{array}$ & $\begin{array}{c}12 \\
(30,0)\end{array}$ & $\begin{array}{c}28 \\
(70,0)\end{array}$ & $\begin{array}{c}40 \\
(3,5)\end{array}$ \\
\hline $40-49$ & $\begin{array}{c}45 \\
(50,6)\end{array}$ & $\begin{array}{c}44 \\
(49,4)\end{array}$ & $\begin{array}{c}89 \\
(10,5)\end{array}$ & $\begin{array}{c}56 \\
(43,1)\end{array}$ & $\begin{array}{c}74 \\
(56,9)\end{array}$ & $\begin{array}{c}130 \\
(11,5)\end{array}$ & $\begin{array}{c}45 \\
(38,8)\end{array}$ & $\begin{array}{c}71 \\
(61,2)\end{array}$ & $\begin{array}{c}116 \\
(10,3)\end{array}$ \\
\hline $50-59$ & $\begin{array}{c}81 \\
(39,7)\end{array}$ & $\begin{array}{c}123 \\
(60,3)\end{array}$ & $\begin{array}{c}204 \\
(24,0)\end{array}$ & $\begin{array}{c}109 \\
(40,8)\end{array}$ & $\begin{array}{c}158 \\
(59,2)\end{array}$ & $\begin{array}{c}267 \\
(23,6)\end{array}$ & $\begin{array}{c}106 \\
(41,4)\end{array}$ & $150(58,6)$ & $\begin{array}{c}256 \\
(22,6)\end{array}$ \\
\hline $60-69$ & $\begin{array}{c}84 \\
(30,9)\end{array}$ & $\begin{array}{c}188 \\
(69,1)\end{array}$ & $\begin{array}{c}272 \\
(32,0)\end{array}$ & $\begin{array}{c}110 \\
(32,4)\end{array}$ & $\begin{array}{c}230 \\
(67,6)\end{array}$ & $\begin{array}{c}340 \\
(30,1)\end{array}$ & $\begin{array}{c}104 \\
(31,2)\end{array}$ & $229(68,8)$ & $\begin{array}{c}333 \\
(29,4)\end{array}$ \\
\hline $70-79$ & $\begin{array}{c}65 \\
(37,4)\end{array}$ & $\begin{array}{c}109 \\
(62,6)\end{array}$ & $\begin{array}{c}174 \\
(20,5)\end{array}$ & $\begin{array}{c}89 \\
(37,7)\end{array}$ & $\begin{array}{c}147 \\
(62,3)\end{array}$ & $\begin{array}{c}237 \\
(21,0)\end{array}$ & $\begin{array}{c}98 \\
(38,7)\end{array}$ & $155(61,3)$ & $\begin{array}{c}253 \\
(22,4)\end{array}$ \\
\hline $80-89$ & $\begin{array}{c}13 \\
(21,0)\end{array}$ & $\begin{array}{c}49 \\
(79,0)\end{array}$ & $\begin{array}{c}62 \\
(7,3)\end{array}$ & $\begin{array}{c}22 \\
(28,9)\end{array}$ & $\begin{array}{c}154 \\
(71,1)\end{array}$ & $\begin{array}{c}76 \\
(6,7)\end{array}$ & $\begin{array}{c}23 \\
(26,1)\end{array}$ & $\begin{array}{c}65 \\
(73,9)\end{array}$ & $\begin{array}{c}88 \\
(7,8)\end{array}$ \\
\hline $90>$ & $\begin{array}{c}0 \\
(0,0)\end{array}$ & $\begin{array}{c}3 \\
(100,0)\end{array}$ & $\begin{array}{c}3 \\
(0,4)\end{array}$ & $\begin{array}{c}0 \\
(0,0)\end{array}$ & $\begin{array}{c}5 \\
(100,0)\end{array}$ & $\begin{array}{c}5 \\
(0,4)\end{array}$ & $\begin{array}{c}1 \\
(10,0)\end{array}$ & $\begin{array}{c}9 \\
(90,0)\end{array}$ & $\begin{array}{c}10 \\
(0,9)\end{array}$ \\
\hline Total & $\begin{array}{c}313 \\
(36,8)\end{array}$ & $\begin{array}{c}537 \\
(63,2)\end{array}$ & $\begin{array}{c}850 \\
(100,0)\end{array}$ & $\begin{array}{c}423 \\
(37,4)\end{array}$ & $\begin{array}{c}708 \\
(62,6)\end{array}$ & $\begin{array}{c}1131 \\
(100,0)\end{array}$ & $\begin{array}{c}410 \\
(36,3)\end{array}$ & $721(63,7)$ & $\begin{array}{c}1131 \\
(100,0)\end{array}$ \\
\hline
\end{tabular}

Tabela III - Prevalência da HAS, por faixa etária, da população com DM de Lajeado-RS, com 15 anos ou mais, cadastrada no SIAB. Lajeado-RS, 2011 a 2013.

\begin{tabular}{lccc}
\hline & $\mathbf{2 0 1 1}$ & $\mathbf{2 0 1 2}$ & $\mathbf{2 0 1 3}$ \\
\cline { 2 - 4 } Faixa etária & $\mathbf{n ~ \%}$ & $\mathbf{n ~ \%}$ & $\mathbf{n ~ \%}$ \\
\hline $15-19$ & $0(0,0)$ & $1(14,3)$ & $1(12,5)$ \\
$20-29$ & $2(11,1)$ & $4(13,8)$ & $4(14,8)$ \\
$30-39$ & $9(37,5)$ & $12(30,0)$ & $14(35,0)$ \\
$40-49$ & $55(61,8)$ & $80(61,5)$ & $68(58,6)$ \\
$50-59$ & $150(73,5)$ & $193(72,3)$ & $184(71,9)$ \\
$60-69$ & $233(85,7)$ & $291(85,6)$ & $285(85,6)$ \\
$70-79$ & $154(88,5)$ & $208(88,1)$ & $224(88,5)$ \\
$80-89$ & $53(85,5)$ & $68(89,5)$ & $81(92,0)$ \\
$90>$ & $3(100,0)$ & $4(80,0)$ & $9(90,0)$ \\
Total & $659(77,5)$ & $861(76,1)$ & $870(76,9)$ \\
\hline
\end{tabular}


A prevalência de gestantes com DM foi de $0,5 \%(n=2)$ em 2012 e de $0,3 \%(n=1)$ em 2013, não havendo casos em 2011.

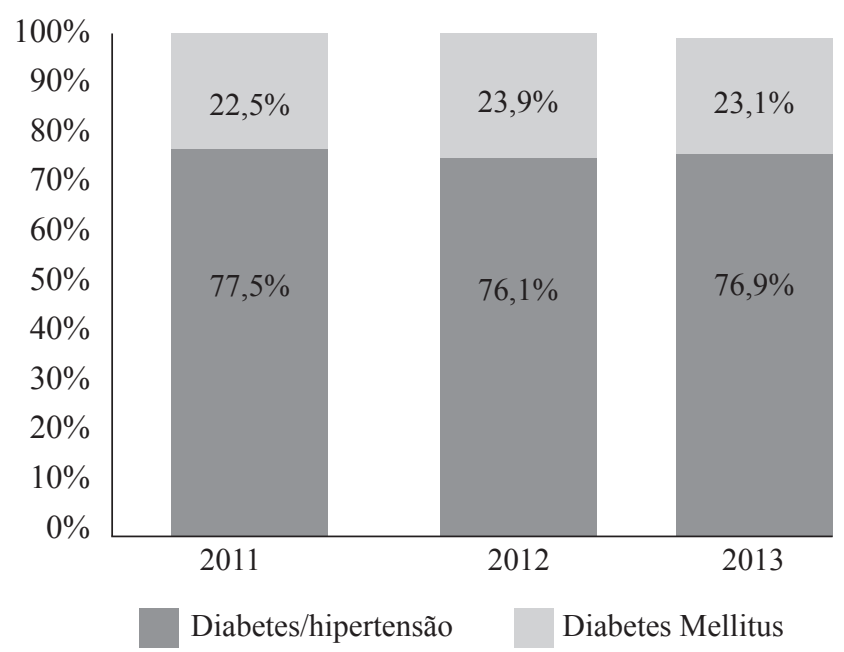

Figura 1 - Prevalência do DM com e sem HAS na população com DM de Lajeado-RS, com 15 anos ou mais, cadastrada no SIAB. Lajeado-RS, 2011 a 2013.

\section{DISCUSSÃO}

Ao analisar a prevalência de DM da população de Lajeado-RS cadastrada no SIAB, nos anos de 2011 a 2013, observou-se que ela está abaixo da média nacional, contudo, manteve-se equiparada nos três anos estudados, isto é, não houve decréscimo na prevalência dessa doença na população estudada. No Brasil, conforme dados epidemiológicos apontados pela pesquisa denominada VIGITEL Brasil, a prevalência de DM foi de 5,6\%, mas em grande parte dessa população a doença não é diagnosticada antes das suas manifestações crônicas terem se estabelecido ${ }^{(3)}$. Outro estudo verificou que a prevalência de DM esteve acima de $10 \%$ na maioria dos estados brasileiros ${ }^{(12)}$.

Um estudo epidemiológico das condições de vida da população de Cuiabá-MT, realizado em 2011 com dados do SIAB, constatou prevalência de DM com $3,21 \%{ }^{(13)}$. Já outro estudo verificou que apenas $1,4 \%$ da população da cidade é acometida por diabetes ${ }^{(14)}$. Em outro estudo, realizado na região do Vale do Taquari-RS, onde está inserido o município de Lajeado-RS, com usuários de Unidades Básica de Saúde (UBS), 15,34\% dos participantes da pesquisa apresentaram glicemia de jejum alterada $(>126 \mathrm{mg} / \mathrm{dl})^{(15)}$.

Quanto à prevalência de alfabetização na população com DM do município de Lajeado-RS, observou-se um percentual elevado de analfabetos. Um estudo realizado no estado de São Paulo constatou que $13 \%$ dos diabéticos são analfabetos ${ }^{(16)}$. Outro estudo realizado com a população adulta do sertão de Pernambuco averiguou que todos os casos de diabéticos estavam entre analfabetos ou indivíduos com apenas o ensino fundamental ${ }^{(17)}$. Também cabe mencionar que o estudo realizado com a população diabética do município de Teixeiras-MG concluiu que $40,9 \%$ dessa população é analfabeta ${ }^{(18)}$. Observa-se que o percentual de pessoas com DM analfabetas de Lajeado-RS ainda é inferior a outros estudos realizados no Brasil ${ }^{(16-18)}$.

A baixa escolaridade pode favorecer a não adesão ao plano terapêutico, pela dificuldade para ler e entender a prescrição, aumentando, assim, os riscos à saúde. Além disso, o baixo nível de escolaridade pode limitar o acesso às informações, devido ao comprometimento das habilidades de leitura, escrita e fala, bem como à compreensão dos complexos mecanismos da doença e do seu tratamento ${ }^{(16)}$. Essa situação traz desafios para a equipe multiprofissional de saúde quanto às estratégias a serem utilizadas para incrementar a adesão ao tratamento dos indivíduos diabéticos ${ }^{(19)}$.

Quanto ao sexo, constatou-se que a prevalência das pessoas com DM no município de Lajeado-RS é maior no sexo feminino (em torno de 63\%), corroborando com outros estudos. Um estudo realizado no estado de São Paulo constatou que houve predomínio do sexo feminino $(69,11 \%)$ na população diabética ${ }^{(16)}$. Em todas as regiões do Brasil, em 2008, a prevalência de diabetes entre mulheres foi maior em comparação com os homens ${ }^{(4)}$. Uma pesquisa que traçou o perfil dos pacientes diabéticos no estado do Pará evidencia que há um predomínio de diabéticos do sexo feminino $(67,08 \%)^{(20)}$.

Neste estudo, a prevalência de diabetes aumentou à medida que aumentava a faixa etária: a partir dos 60 anos de idade, há um grande aumento na prevalência de diabetes. Um estudo que traçou o perfil sociossanitário e o estilo de vida da população diabética do município de TeixeirasMG também encontrou uma predominância de indivíduos idosos, sendo que a média de idade foi de $63,5( \pm 13,12)$ (18). No Brasil, dados da VIGITEL, de 2011, mostram que a prevalência de DM aumenta de acordo com a idade da população: 21,6\% dos brasileiros com mais de 65 anos referiram a doença, um índice bem maior do que entre as pessoas na faixa etária entre 18 e 24 anos, em que apenas $0,6 \%$ são pessoas com diabetes ${ }^{(3)}$.

Quanto à prevalência da HAS nas pessoas com DM, observou-se que o percentual ficou em torno de $77 \%$, havendo um aumento nos três anos estudados. Constatou-se que a maioria da população diabética é hipertensa. A prevalência de diabetes e hipertensão associada está crescendo rapidamente no $\mathrm{Brasil}^{(4)}$. Um estudo que investigou a prevalência de DM, sua relação com características sociodemográficas e outros fatores de risco cardiovascular em uma população adulta do sertão 
de Pernambuco, constatou que 68,0\% dos diabéticos eram hipertensos $^{(17)}$.

Ao analisar a prevalência da HAS por faixa etária na população com DM em Lajeado-RS, verificou-se que, nos anos de 2012 e 2013, houve casos de diabéticos hipertensos desde a faixa etária de 15 a 19 anos. A hipertensão arterial na população jovem está aumentando devido a mudanças na rotina de vida, como nos hábitos alimentares e o sedentarismo, que podem levar à obesidade, sendo considerados fatores de risco para a hipertensão. Além disso, há falta de acompanhamento médico por parte dos jovens, haja vista que, dentre estes, poucos se preocupam em realizar avaliações médicas periódicas ${ }^{(21)}$.

Na variável "prevalência de gestantes com diabetes", constatou-se que, em 2012 e 2013, houve baixa prevalência (0,5\% e 0,3\%), com ausência de casos em 2011. Novamente, salienta-se que os dados são autorreferidos e pode haver gestantes com DM gestacional não diagnosticada. O DM gestacional é o problema metabólico mais comum na gestação e tem prevalência entre $3 \%$ e $13 \%$ das gestações. A prevalência estimada dessa doença no Brasil é de 7,6\% entre as gestantes com mais de $20 \operatorname{anos}^{(22)}$. Um estudo que avaliou a prevalência de diabetes em gestantes de VitóriaES verificou prevalência de 5,8\%(23)

Cabe destacar a importância de se realizar pesquisas que avaliem a prevalência de doenças crônicas como o diabetes, visto que, além de possibilitar o conhecimento da população que possui a doença, também pode contribuir para o planejamento de políticas públicas e delineamento de estratégias de saúde visando a promoção da saúde ${ }^{(24)}$.

Acredita-se que este estudo pode apresentar como limitação o fato de ter sido realizado com pessoas que se autorreferem possuir DM. Tal situação pode contribuir para um baixo percentual encontrado desta população, pois o DM é uma doença que, muitas vezes, permanece assintomática e, consequentemente, algumas pessoas possuem DM, mas desconhecem a sua existência.

Considera-se também que pode ser outra limitação do estudo o fato de o SIAB não contemplar todas as pessoas que residem no município de Lajeado-RS. Segundo o IBGE, a população geral com 15 anos ou mais do município, em 2011, foi de 64.470; destes, 44,7\% $(n=28.794)$ indivíduos foram cadastrados no SIAB. Em 2012, a população geral com 15 anos ou mais foi de 64.083 e foram cadastrados $57,2 \%(n=36.662)$ indivíduos. Já em 2013, a população de Lajeado-RS com 15 anos ou mais era de 67.397, sendo $55,6 \%(n=37.495)$ indivíduos cadastrados no SIAB.

\section{CONCLUSÃO}

Observou-se que a prevalência das pessoas com DM, autorreferidos, com 15 anos ou mais, cadastrados no SIAB do município de Lajeado-RS, de 2011 a 2013, manteve-se equiparada nos anos de 2011 a 2013, encontrando-se abaixo da média nacional. Verificou-se que essa população, na sua maioria, está acima de 60 anos, é alfabetizada (embora com elevado percentual de analfabetos), feminina, hipertensa, com baixa prevalência de gestantes com diabetes. Essas características são importantes para a gestão municipal planejar ações de promoção da saúde adequadas à população.

\section{AGRADECIMENTOS}

À Fundação de Amparo à Pesquisa do Estado do Rio Grande do Sul (FAPERGS), pela concessão de bolsa de doutorado para a primeira autora do presente estudo.

\section{REFERÊNCIAS}

1. O'keefe JH, Bell DSH, Wyne, KL. Fundamentos em diabetes. $4^{\mathrm{a}}$ ed. Porto Alegre: Artmed; 2010.

2. International Diabetes Federation. IDF Diabetes Atlas. $6^{\text {th }}$ ed. Brussels: International Diabetes Federation; 2013 [acesso em 2014 Abr 22]. Disponivel em: http:// www.idf.org/sites/default/files/EN_6E_Atlas_Full_0. pdf

3. Ministério da Saúde (BR), Secretaria de Vigilância em Saúde, Secretaria de Gestão Estratégica e Participativa. Vigitel-Brasil 2011: vigilância de fatores de risco e proteção para doenças crônicas por inquérito telefônico. Brasília: Ministério da Saúde; 2012.

4. Freitas LRS, Garcia LP. Evolução da prevalência do diabetes e deste associado à hipertensão arterial no Brasil: análise da Pesquisa Nacional por Amostra de Domicílios, 1998, 2003 e 2008. Epidemiol Serv Saúde. 2012;21(1):7-19.

5. Ministério da Saúde (BR), Secretaria de Atenção à Saúde, Departamento de Atenção Básica. Estratégias para o cuidado da pessoa com doença crônica: diabetes mellitus. Brasília: Ministério da Saúde; 2013.

6. Schmidt MI, Duncan BB, Stevens A, Luft V, Iser BPM, Moura L, et al. Doenças crônicas não transmissíveis no Brasil: mortalidade, morbidade e fatores de risco. In: Ministério da Saúde (BR). Saúde Brasil 2009: uma análise da situação de saúde e da Agenda Nacional e Internacional de Prioridades em Saúde. Brasília; 2010. p. 111-36.

7. Duarte MLC, Tedesco JR, Parcianello RR. O uso do sistema de informação na estratégia saúde da família: percepções dos enfermeiros. Rev Gaúcha Enferm. 2012;33(4):111-7. 
8. Carreno I, Moreschi C, Marina B, Hendges DJB, Rempel C, Oliveira MMC. Análise da utilização das informações do Sistema de Informação de Atenção Básica - SIAB: uma revisão integrativa. Ciênc Saúde Coletiva. 2015; 20(3):947-56.

9. Silveira JAA, Resende HMP, Lucena FAM, Pereira JG. Características da assistência à saúde a pessoas com diabetes mellitus acompanhadas na Unidade de Saúde da Família Pedregal II, em Cuiabá, MT: reflexões para a equipe de saúde. Mundo Saúde. 2010;34(1):43-9.

10. Instituto Brasileiro de Geografia e Estatística - IBGE. XII Censo Demográfico [Internet]. 2010 [acesso em 2014 Mar 12]. Disponível em: http://www.ibge.gov.br/ home/estatistica/populacao/censo2010/

11. Instituto Brasileiro de Geografia e Estatística - IBGE. XII Censo Demográfico [Internet]. 2013 [acesso em 2014 Mar 12]. Disponível em: http:www.ibge.gov.br

12. Dias JCR, Campos JADB. Diabetes mellitus: razão de prevalências nas diferentes regiões geográficas no Brasil, 2002 - 2007. Ciênc Saúde Coletiva. 2012;17(1):239-44.

13. Silva MCN, Ávilla AL, Silva BPS, Alves LS, Santos DS, Rafael JC. Perfil epidemiológico e social da população atendida em uma Unidade Básica de Saúde em Cuiabá. Gestão \& Saúde. 2013;4(2):25-37.

14. Silva LM. Prevalência de diabetes melito e fatores associados em população urbana adulta de baixa escolaridade e renda do sertão nordestino brasileiro. Arq Bras Endocrinol Metab. 2010;54(6):560-6.

15. Rempel C, Strohschoen AG, Hoerlle JL, Sartori MAB, Busch GC, Périco E, et al. Perfil dos usuários de Unidades Básicas de Saúde do Vale do Taquari: fatores de risco de diabetes e utilização de fitoterápicos. ConScientiae Saúde. 2010;19(1):17-24.

16. Rodrigues FFL, Santos MA, Teixeira CRS, Gonela JT, Zanetti ML. Relação entre conhecimento, atitude, escolaridade e tempo de doença em indivíduos com diabetes mellitus. Acta Paul Enferm. 2012;25(2):28490.

17. Lyra R, Silva RS, Montenegro RMJ, Matos MVC, Cézar NJB, Silva LM. Prevalência de diabetes melito e fatores associados em população urbana adulta de baixa escolaridade e renda do sertão nordestino brasileiro. Arq Bras Endocrinol Metab. 2010;54(6):560-6.

18. Cotta RMM, Batista KCS, Reis RS, Souza GADG, Dias G, Castro FAF, et al. Perfil sociossanitário e estilo de vida de hipertensos e/ou diabéticos, usuários do Programa de Saúde da Família no município de Teixeiras, MG. Ciênc Saúde Coletiva. 2009;14(4):1251-60.

19. Xavier ATF, Bittar DB, Ataide MBC. Crenças no autocuidado em Diabetes: implicações para a prática. Texto \& Contexto Enferm. 2009;18(1):124-30.

20. Palmeira MM, Silva LL, Sousa RM, Cordeiro CRG. Perfil epidemiológico dos pacientes diabéticos atendidos no Serviço de Diagnóstico em Cardiologia da Universidade do Estado do Pará. RBM Rev Bras Med. 2011;11(2):29-32.

21. Vasconcelos KNC, Silva JCL, Groppo FC, Bergamaschi CC, Cabral LN, Athayde MMS. Prevalência de jovens hipertensos não diagnosticados atendidos na policlínica odontológica da Universidade do Estado do Amazonas. Odonto. 2012;20(39):89-98.

22. Ministério da Saúde (BR), Secretaria de Atenção à Saúde, Departamento de Atenção Básica. Atenção ao pré-natal de baixo risco. Brasília: Ministério da Saúde; 2012.

23. Massucatti LA, Pereira RA, Maioli TU. Prevalência de diabetes gestacional em unidades de saúde básica. Rev Enferm Atenção Saúde. 2012;1(1):70-9.

24. Pozzobon A, Hoerlle JL, Carreno I. Prevalência de diabetes e hipertensão em indivíduos cadastrados no sistema de informação da atenção básica (SIAB) de Lajeado/RS- Brasil. Rev Bras Promoç Saúde. 2014;27(3):295-302.

\section{Endereço para correspondência:}

Claudete Moreschi

Rua Avelino Tallini, 171

Bairro Universitário

CEP: 95900-000 - Lajeado - RS - Brasil

E-mail: clau_moreschi@yahoo.com.br 